\title{
Real Time Synthetic Aperture and Plane Wave Ultrasound Imaging with the Xilinx VERSALTM SIMD-VLIW Architecture
}

\author{
Corradi , Giulio ; Jensen, Jørgen Arendt
}

Published in:

2020 IEEE International Ultrasonics Symposium

Link to article, DOI:

10.1109/IUS46767.2020.9251749

Publication date:

2020

Document Version

Peer reviewed version

Link back to DTU Orbit

Citation (APA):

Corradi , G., \& Jensen, J. A. (2020). Real Time Synthetic Aperture and Plane Wave Ultrasound Imaging with the Xilinx VERSAL TM SIMD-VLIW Architecture. In 2020 IEEE International Ultrasonics Symposium IEEE.

https://doi.org/10.1109/IUS46767.2020.9251749

\section{General rights}

Copyright and moral rights for the publications made accessible in the public portal are retained by the authors and/or other copyright owners and it is a condition of accessing publications that users recognise and abide by the legal requirements associated with these rights.

- Users may download and print one copy of any publication from the public portal for the purpose of private study or research.

- You may not further distribute the material or use it for any profit-making activity or commercial gain

- You may freely distribute the URL identifying the publication in the public portal

If you believe that this document breaches copyright please contact us providing details, and we will remove access to the work immediately and investigate your claim 


\section{Real Time Synthetic Aperture and Plane Wave Ultrasound Imaging with the Xilinx VERSAL ${ }^{\text {TM }}$ SIMD-VLIW Architecture}

\author{
Giulio Corradi \\ Healthcare \& Sciences \\ Xilinx Inc. \\ San Jose California USA \\ giulio.corradi@xilinx.com
}

\author{
Jørgen Arendt Jensen \\ Technical University Denmark \\ Lyngby, Denmark \\ jaje@dtu.dk
}

\begin{abstract}
A decade of technological improvement in SystemOn-Chip (SoC) integrated with Field Programmable Gate Arrays (FPGAs) have made it possible to successfully productize traditional ultrasound systems, especially portable ones. However, advanced modalities like Synthetic Aperture (SA) and Plane Wave (PW) Ultrafast imaging with their many advantages have not been targeted because of the sheer computing performance required for the ultrasound pipelines in real-time. Very large vector processers (DSPs and GPUs) solves some of the problems, but it runs into traditional scaling challenges due to inflexible, inefficient memory bandwidth usage and high-power consumption. Traditional FPGA solutions provide a programmable memory hierarchy, but the hardware development flow has been a barrier to broad, highvolume adoption. The need for low power and high performance must be explored beyond the conventional "one size fits all" CPU scalar processing solution for Ultrafast imaging to be a viable option. This paper presents a new technology called 'Adaptive Compute Acceleration Platform' (ACAP) on Xilinx's Versal' system, which has the capability of making real time SA and PW Ultrafast imaging possible.
\end{abstract}

Keywords - ultrasound, plane wave, synthetic aperture, image reconstruction, FPGA

\section{INTRODUCTION}

Plane Wave (PW) and Synthetic Aperture (SA) are well studied techniques that significantly improve frame rate enabling ultrafast at thousands of frames per second. The working principle of PW and SA is similar. Echoes are recorded using all elements on the receive aperture. The acquired data set is complete to reconstruct a low-resolution full image (LRI). Image, focus is possible in both transmit and receive. However, LRI image has poor contrast and need quality improvement. Repeating transmit-receive process and acquiring other LRIs images for a predetermined number of emissions and combining with the proper phase alignment all the LRI it gives a highresolution image (HRI).

PW and SA increase also the image quality in-vivo and increase penetration depth [2, 10]. It can follow moving structures and blood for all time and in all directions [11]. It can increase the precision of velocity estimates by a factor of ten, and estimation of the velocity vector [12]. The ultrafast frame rates allow detection of tissue elasticity and perform shear wave imaging [13].

Designing an ultrasound system for real-time SA and PW is very challenging because the sheer amount of data produced per second requires very high computing performance that limited their implementation on commercial systems.

Moreover, for a practical design, it is important to keep as integrated as possible the Front End Electronic (FE) to minimize the latency, and to provide fast data access to the image processing pipeline for real-time images.

Recent development in the semiconductor technology reaching $7 \mathrm{~nm}$ of transistor size can enable the design of advanced ultrasound systems based on heterogeneous systemon-chip with adequate performances. This paper explores the application of this heterogeneous system on chip technology using the VersalTM device produced by Xilinx Inc.

\section{PW AND SA ALGORITHMS}

To understand the process of LRI and its compounding, the HRI equation can be written as:

$$
H R I\left(\vec{r}_{p}\right)=\sum_{j=1}^{N x d c} \sum_{i=1}^{N E} a\left(t_{p}(i, j), i, j\right) y_{r}\left(t_{p}(i, j), i, j\right)
$$

Where $\vec{r}_{p}$ is the geometric distance from the emitting element to the imaging point, $p=1,2,3, \ldots, P$ the current point in the set of $P$ points, $y_{r}\left(t_{p}(i, j), i, j\right)$ is the received signal from emission $i$ on element $j, a\left(t_{p}(i, j), i, j\right)$ is the apodization function, $N x d c$ is the number of transducer elements, $N E$ the number of emissions.

The parameter $t_{p}(i, j)$ is the time for transmission and reception. For synthetic aperture the delay is function of position of the transmitting element and function of position of the receiving element $j$.For PW the unfocused nature of the emission requires also the direction of propagation.

Direction of propagation allows quality improvement tuning the angle of increment between emissions.

Resolution of the $t_{p}(i, j)$ delay determines the quality of the beamforming but it requires fractional samples. Interpolation 
recovers the fractional samples but it is very computational demanding. Four interpolations are considered: linear interpolation, sampling frequency increased by a factor of 10 combined with linear interpolation (interp), spline interpolation and piecewise cubic Hermite interpolating polynomial. Proper side lobes are only attained for the spline and for the interp function and are those selected for this work.

\section{A. Spline interpolation complexity}

Catmull-Rom Splines (CRS) is evaluated for complexity because it has continuity properties and is widely used also in computer graphic. Twelve multiplications and ten additions/subtractions are required for every interpolated point, that must be executed for thousands of points. Thus, the spline algorithm is considered but not further implemented in the scope of this paper.

\section{B. Matched filter interpolation}

For improving the data reuse and the efficiency it is possible to use as interpolator the matched filter already needed in the receiver stage. The interpolation is achieved by increasing the sampling frequency by a factor $\mathrm{D}$. The matched filter has a length 20 samples when $D=4$, this is also the number of multiply/add to perform for the interpolation. Side lobes are lower than for interp interpolation, but axial resolution is slightly worse, however this is an acceptable trade-off for the extent of this work.

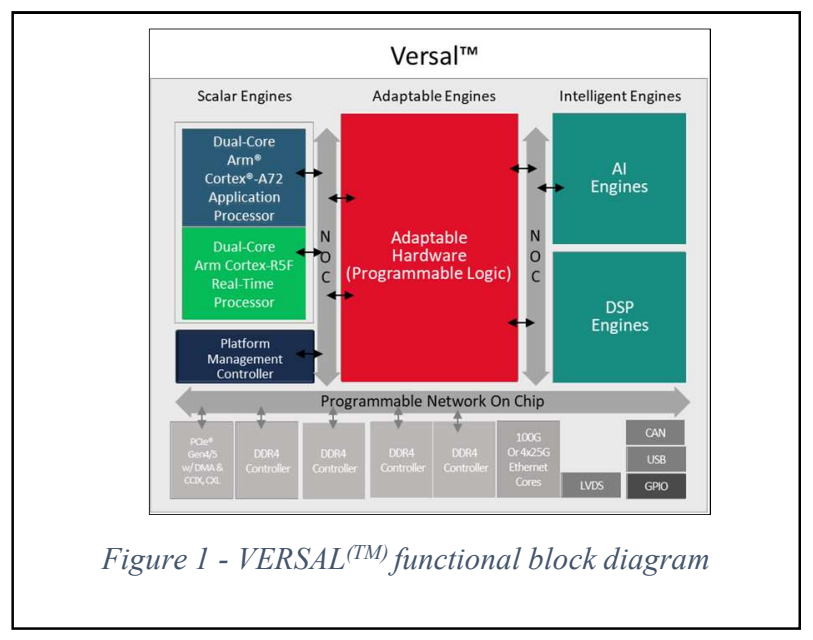

\section{Compounding for HRIs}

Similarly, for compounding the HRIs, its process involves multiple computations of same set of data, however because the equation $(1)(1)$ is linear there is a way to express it recursively employing the idea of "sliding window" to reduce the calculations [1]. The last emission is added, and the first emission is subtracted. This approach takes advantage of data reuse that will be exploited in the Versal ${ }^{\mathrm{TM}}$ architecture.

\section{VERSAL ${ }^{\mathrm{TM}}$ ARCHITECTURE}

Most of the computation required for an ultrasound scanner rely heavily on vectors (e.g. scan lines, RF data, etc.), matrices (e.g. apodization weights, delays, interpolation kernels, etc.), and the combination of vectors, and matrices with the emissions can be translated easily into tensors, taking advantage of the compact representation that tensors allows. To extract the highest frame rate, all the operations listed above need to be parallelized as much as possible. Vector processors like the ones available in GPUs can be used for executing the parallel computation but they lack the direct connectivity to the transducers. In case of GPUs RF data that need to be acquired separately, stored in intermediate memory on a PC, and transferred using PCle that even if it is very fast introduces complications and latency. Versal ${ }^{\mathrm{TM}}$ instead offers all the interfaces for the frontend, the computational elements, the communication ports, and the memory control all in a single device, allowing a very high integration.

\section{A. Hardware Setup}

The selected Versal ${ }^{\mathrm{TM}}$ System-On-Chip (SOC) [6] integrates a dual-core Arm ${ }^{\circledR}$ Cortex ${ }^{\circledR}-\mathrm{A} 721 \mathrm{GHz}$ (APU) and a dual-core Arm Cortex-R5F 750MHz (RPU), called by Xilinx commercial nomenclature a scalar processing system (PS). PS includes several peripherals for communication standards, including gigabit Ethernet, USB, and controllers for SPI, I2C, UART, and CAN-FD, and others.

Four DDR4 memory controllers are available in the chip, partitioned into 1 for the PS program and data storage, used by both A72 and R5F, and 3 DDR4 for storing and retrieving the RF data. Each DDR4 allows a data rate of 25.6 GByte/s. For this work only two controllers used, one for PS and one for the RF.

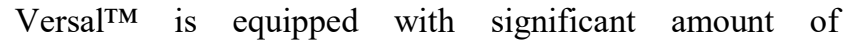
programmable hardware, normally known as FPGA identified in the chip as adaptable hardware. It provides many functions like memory, analog blocks and high speed I/O that were uncommon in previous generations. The adaptable hardware is used for managing the receive and transmission logic at the sampling and oversampling frequencies. It includes JESD204C [3] [4] [5] compatible interface block supporting line rates from $1 \mathrm{~Gb} / \mathrm{s}$ to $32 \mathrm{~Gb} / \mathrm{s}$. The receive stage for this work targets the TI AFE58JD2 chips with 16 channels AFE providing 12 bit at a sampling frequency of 80 Mega Samples Per Second (MSPS) per channel with JESD204C connectivity, but LVDS is also possible. Versal ${ }^{\mathrm{TM}}$ provides both interfaces.

The adaptable hardware logic implements smart data movers to offload the real-time information from and to DDR4 and to any of the specific subsystem. The data movers are fundamental building blocks to provide the necessary bandwidth for the sampled and elaborated data.

To expand the acquisition stage for more channels in a modular fashion, a $10 \mathrm{Gbit} / 25 \mathrm{Gbit}$ Ethernet transparent switch for real-time streaming of data is possible. For example, if 128 channels are desired, the 2-port transparent switch capability allows the modules to be daisy chained. UDP/IP protocol provides the data exchange reducing the amount of wires, and it is accelerated in hardware, including Precision Time Protocol (PTP) IEEE 1588 for timestamping to achieve synchronised clock accuracy in the sub-microsecond range.

In the same logic, there are 1,968 Digital Signal Processors (DSP) engines capable of $27 \times 24$ two's complement multiply 
and 58-bit accumulator (MAC) at $1 \mathrm{GHz}$, complex multiplyaccumulator at $950 \mathrm{MHz}$ and single-precision floating-point (32SPFP) with 32-bit accumulation at $800 \mathrm{MHz}$.

Part of PW and SA algorithms is executed with such DSPs specifically for the front end, the demodulation, and filtering.

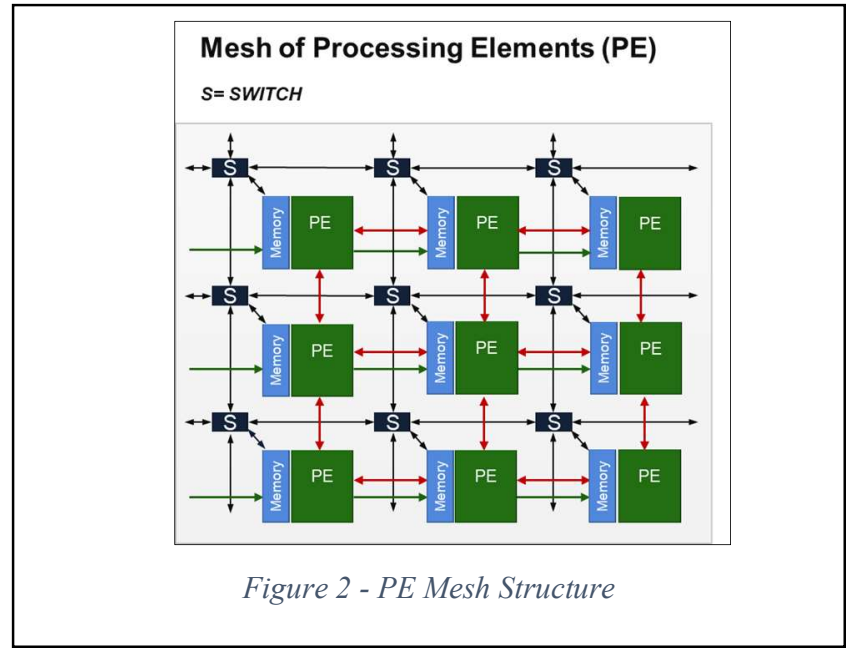

\section{B. Intelligent Engine}

The intelligent engine [8] is the main computational unit for the whole PW and SA. It is fed by the programmable logic that implements the data movers. The engine implements an architecture that is a set of homogeneous processors elements (PE) in a tessellated (tiles) structure with a collection of a switch lattice, to connect PEs. It forms a regular structure of programmable switches connected by data paths. Provide local exploitation so that data movement is bounded to adjacent processing elements. Provides pipelining used to achieve high PE utilization.

It is organized in a mesh of tiles. Each tile contains a Very Long Instruction Word (VLIW) processors. The VC1902 hosts 400 PEs. The PW and SA algorithms are decomposed in functional elements, and every functional element is expressed as set of elementary operations executing into one or more PEs pipelined with a graph that chains all the operations.

Each step of PW and SA algorithm is decomposed into the set of functional elements and elementary operations with $\mathrm{C}++$ templates creating a pipeline process allowing concurrency.

The mesh structure of the intelligent engines is shown in Figure 2. Arrows are the interconnects of the PE to other PEs, to programmable logic and to DDR memory. The block $\mathrm{S}$ is a communication switch that allows each PE to form a logical graph. The communication path is managed setting the route in the switches. The dataflow can be executed horizontally, vertically or diagonally as appropriate for the specific functional unit algorithm. To minimize the data fetch from the main DDR, that adds overhead, each PE has a local $32 \mathrm{Kbyte}$ fast memory that is used as cache or as shared buffer for the next PE.

The PE can execute operations in single instruction multiple data single instruction multiple data (SIMD) with a word length of 512 bit. The parallelism for every 16 bit operands is 32 , this means that 32 multiplication-accumulate are executed per instruction. For every floating point instruction, the parallelism is 8 .

Every PE runs at $1 \mathrm{GHz}$ and RF data is normalized as a 16bit integer, the data sampled is 12 bits. After the Hilbert transform, the data is 16-bit complex, other operations are in floating point. The PE supports natively all such operations allowing at least parallelism 16 for real integer data and complex and parallelism 4 for complex integer data vs complex, that's for each tile.

\section{Network on Chip NoC}

A fundamental role is played by the programmable network on chip (NoC). It is an interconnecting network used for sharing data between modules endpoints in the programmable logic, the processing system (PS), and intelligent engine AI, and the DDR4 memories. It consists of a series of horizontal paths (HNoCs) and vertical paths (VNoCs). The NoC is configured at boot to connect the Receiver and Transmitter control path to the A72 processors in the PS, to connect the Receiver RF acquisition stream from the front-end filters into the storage DDR4, to connect the Transmitter sequences stored in memory into the Transmitter module, to push the stored RF data into the intelligent engine. Think of the $\mathrm{NoC}$ as a highway within the device responsible to guarantee the proper traffic among blocks.

The full description of Versal ${ }^{\mathrm{TM}}$ can be found in the documents available in [7].

\section{PERFORMANCE TARGET}

For the validation of the proposed architecture the AI engine is the main computational unit, aided by programmable logic for filtering and data movement. All the functions are orchestrated by the A72 cores. The performance target for this paper, deals with PW investigation of $150 \mathrm{~mm}$ depth using 64 elements. Parameter study has been conducted calculating the lowresolution images to see the effects on the PSF. At 7 plane waves the resulting PSF is acceptable and it is thus possible to target an imaging system with a frame rate of $5040 / 7=720$ frames/sec enough for a realistic situation where the chip is properly exploited.

Here 96 PEs are used for integer calculations and 206 for floating point single precision calculations. The total utilization ratio of the AI engine is $76 \%$ and DSPs resources at $50 \%$ of the ones available in the PL. Latency is determined by the slowest 
block in the pipeline and it is currently around 41 microseconds (us). This setup allows the achievement of the desired target.

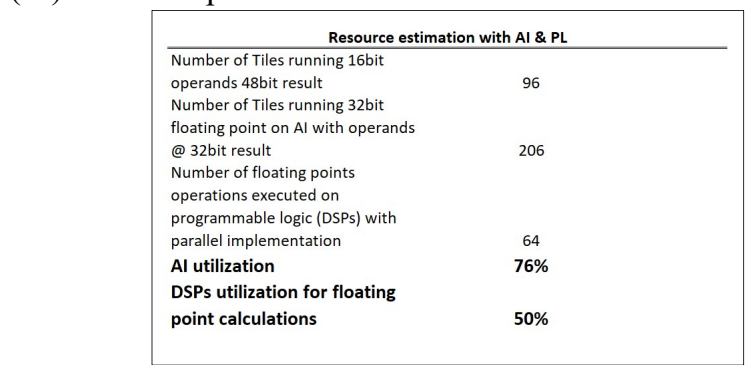

Figure 3 - Resource utilization for the PW HRI with 7 emissions 64 elements@720 Hz and $5 \mathrm{KHz}$ repetition

\section{SOFTWARE IMPLEMENTATION}

The system runs Linux on the A72 pair allowing control of the $\mathrm{AI}$ engine. A combination of Python and $\mathrm{C}++$ are used as main development programs running natively in the $\mathrm{VC} 1902$ chip. Numpy [9] is used for the management of the off-line data manipulation and visualization with Jupyter Notebook [16] all hosted locally in the chip. Only a HTML browser connected to the $1 \mathrm{Gbit}$ Ethernet port is the main interface. Clearly this setup is used for the development phase and performance evaluation, and it is not intended for a commercial product. However, the full infrastructure is ready for being integrated into a commercial scanner. To validate the system in hardware, because the transducers are not available at the time of this publication, a database generated with Field II has been prepared with a group of phantoms located at different depths, from $50 \mathrm{~mm}$ to $150 \mathrm{~mm}$. The files produced by Field II are stored into one DDR4 memory and fetched as acquired by the front-end transducers, emulating the real transducers.

The algorithms have been described accordingly to the AI engine dataflow using the Xilinx Vitis ${ }^{\circledR}$ framework Figure 4 shows a portion view of the graph.

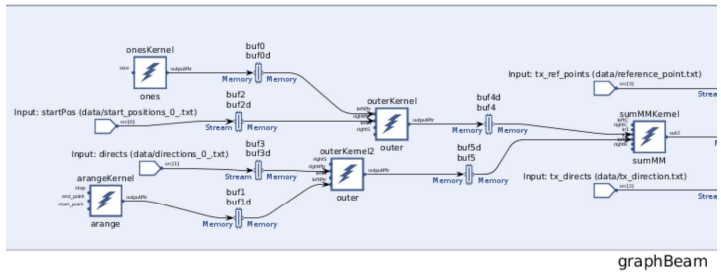

Figure 4 - SA and PW beamforming graph detail

For this implementation, there are 34 kernels executed on every tile in a group of 302 tiles, while a portion of the calculation is executed in the PL for the filtering, including the data mover which is realized in the PL. Figure 3 shows the total resources used between the AI engine and programmable hardware for 720 frames/s.

\section{CONCLUSION AND FUTURE WORK}

This paper shows that Versal ${ }^{\mathrm{TM}}$ system on chip can execute PW and SA algorithms autonomously as close as possible to the transducer at a rate of $720 \mathrm{frames} / \mathrm{s}$. Such frame rate is in general achievable only using more complex setup, often using multiple
GPUs and FPGA for the front end. The main benefit of this approach compared to traditional full FPGA or full GPU is that there is a higher integration and a lower latency. The selected PW setup for the analysis is a trade-off between frame rate and quality of the PSF, however the amount of computation is adequate for providing images of good quality. Additionally, even if not completely described in this paper to keep it focused on PW and SA, the infrastructure for communication, synchronization and modularity is an architectural plus. It shows that it is possible to realise an extensible scanner architecture with a reduced infrastructure. The proposed approach is probably suitable also for research system because is quickly extendable.

\section{REFERENCES}

[1] Nikolov, S., Gammelmark, K., \& Jensen, J. A. (1999). Recursive Ultrasound Imaging. In 1999 IEEE Ultrasonics Symposium Proceedings (Vol. 1-2, pp. 1621-1625). IEEE. IEEE International Ultrasonics Symposium.Proceedings https://doi.org/10.1109/ULTSYM.1999.849306

[2] Gammelmark, K., \& Jensen, J. A. (2003). Multielement Synthetic Transmit Aperture Imaging Using Temporal Encoding. IEEE Transactions on Medical Imaging, 22(4), 552-563. https://doi.org/10.1109/TMI.2003.809088

[3] JESD204C Standard www.jedec.org

[4] JESD204 PHY LogiCORE IP Product Guide (PG198 v4.0 June 03, 2020) https://www.xilinx.com/support/documentation/ip documentation/jesd2 04_phy/v4_0/pg198-jesd204-phy.pdf

[5] JESD204C v4.2 Product Guide (PG242 v4.2 July 16, 2020) https://www.xilinx.com/support/documentation/ip_documentation/jesd2 04c/v4 2/pg242-jesd204c.pdf

[6] Versal: The First Adaptive Compute Acceleration Platform (ACAP) (WP505 v1.0.1 September 23, 2019) https://www.xilinx.com/support/documentation/white papers/wp505versal-acap.pdf

[7] Versal ${ }^{\mathrm{TM}}$ AI Core - website https://www.xilinx.com/products/silicondevices/acap/versal-ai-core.html\#documentation

[8] Versal ACAP AI Engine Architecture Manual (AM009 v1.0 July 16, 2020) https://www.xilinx.com/support/documentation/architecturemanuals/am009-versal-ai-engine.pdf

[9] Numpy https://numpy.org/

[10] Pedersen, M. H., Gammelmark, K. L., \& Jensen, J. A. (2007). In-vivo evaluation of convex array synthetic aperture imaging. Ultrasound in Medicine \& Biology, 33(1), 37-47. https://doi.org/10.1016/j.ultrasmedbio.2006.07.041

[11] I. S. Jacobs and C. P. Bean, "Fine particles, thin films and exchange anisotropy," in Magnetism, vol. III, G. T. Rado and H. Suhl, Eds. New York: Academic, 1963, pp. 271-350.

[12] M. Tanter, J. Bercoff, L. Sandrin, and M. Fink, "Ultrafast compound imaging for 2-D motion vector estimation: application to transient elastography,"IEEE Trans. Ultrason., Ferroelec., Freq. Contr., vol. 49,pp. 1363-1374, 2002

[13] E. Mace, G. Montaldo, I. Cohen, M. Baulac, M. Fink, and M. Tanter, "Functional ultrasound imaging of the brain," Nature methods, vol. 8,no. 8, pp. 662-664, 2011

[14] J. A. Jensen and N. B. Svendsen, "Calculation of pressure fields from arbitrarily shaped, apodized and excited ultrasound transducers," ", IEEE Trans. Ultrason., Ferroelectr., Freq. Control, vol. 39, no. 2, pp. 262-267, March 19921992.

[15] J. A. Jensen, "Field: A Program for Simulating Ultrasound Systems," Medical \& Biological Engineering \& Computing, vol. 34, Supplement 1, Part 1, pp. 351-353, 1996.

[16] Jupyter Notebook https://jupyter.org/ 